\title{
Conceit for system of Self-rated health measurement and establishment exercise prescription based on Artifical Neural Network and Expert System
}

\author{
Qiang LI \\ Department of Physical education, North-west University, Xi'an, 710069, China \\ 23414388@qq.com
}

Keywords: Self-rated health measurement, Exercise prescription, Artifical Neural Network, Expert System

\begin{abstract}
People pursue higher quality life than previously along with development of society. Quality of life decided level of health. How to estimate oneself health and exercise is one of the heatedly discussed topics. According to the theory of Artifical Neural Network and Expert System, A design conceit for system of Self-rated health measurement and establishment exercise prescription based on Artifical Neural Network and Expert System. In order to serve as reference for everyone of pursuing health .
\end{abstract}

\section{Introduction}

Exercise prescription is the arrangement of suitable type of sports, duration and frequency, in the form of prescription, according to the medical data (including sport experiments and physical test), and the conditions of health, physique, cardiovascular and relevant living environment and hobbies. Also, it can point out the matters need attention during sports so as to make the excises more regularly and acquire better body building and sick cure.

With the development of the society, people have higher quality of life (QOL). The health level is a core factor that influence a people's QOL. Nowadays, more and more people realize the importance of physical exercises. Therefore, how to precisely evaluate the health condition in real time and then adapt to a suitable sport plan is currently a popular problem among us. Here, we propose a system that help people self-assess the healthy level and make sport plans, based on artificial neural networks (ANNs) and expert system.

\section{Assumption of Health Evaluation and Prescription Constitution System Based on ANNs}

\section{Introduction to ANNs}

Artificial neural networks (ANNs) is the complex networks that consist of a large number of simple nodes (neurons), which interconnect with each other. It is the simplification, abstraction and simulation of human brain. So far, there are hundreds of neuron models reported by references[1].

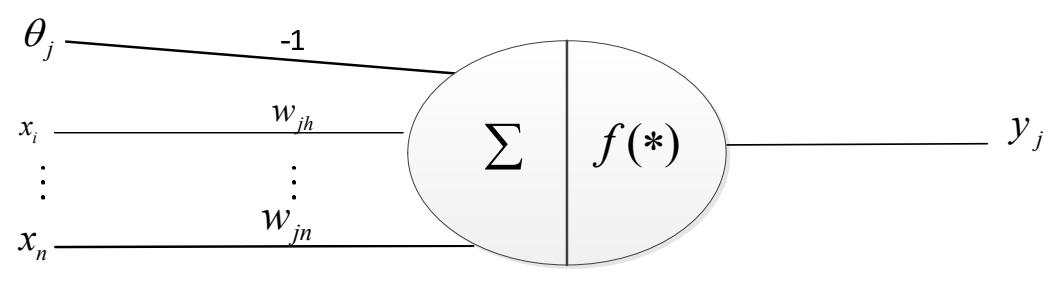

Fig. 1 Artificial Neuron model

$\theta_{j}$ - threshold; ${ }^{x_{i}}$-input; ${ }^{w_{j i}}$ - connecting weight; -Output transformation function; ${ }^{y_{j}}$ Output 
Figure 1 shows a typical and common ANN model. It has a parallel and a single output, which connect with many other neurons. The input has many pathways, which corresponds with a weight coefficient. This relationship of input and output can be shown as (in mathematical form)[2].

$$
\begin{aligned}
& \mathrm{S}_{\mathrm{j}}=\sum_{i=1}^{n} \mathcal{W}_{\mathrm{ji}} \mathrm{X}_{\mathrm{i}}^{-} \theta_{\mathrm{j}}=\sum_{i=o}^{n} \mathcal{W}_{\mathrm{ji}} \mathrm{X}_{\mathrm{i}} \quad\left(\mathrm{x}_{0}=\theta_{\mathrm{j}}, \quad \mathrm{w}_{\mathrm{j} 0}=-1\right) \\
& \mathrm{y}_{\mathrm{i}}=f^{\text {(sj) }}
\end{aligned}
$$

where $\mathrm{Sj}$ is the summation of each input variables (linear combination).

The working process of an ANN consists of two stages. The first stage is the working period. At this moment, the weight coefficients are fixed. The calculation units' statuses change in order to reach a stable stage. The other stage is the learning stage. At this moment, the statuses of calculation units are fixed. The weight coefficients change according to the learning pattern of samples.

\section{Health Evaluation and Prescription Constitution System Based on ANNs}

Structure of ANNs. The self-assessment and prescription constitution are expressed by ANNs. Aiming at making health evaluation and prescription constitution for people in different culture background, social structure and sense of worth, we need to develop a complex ANN system which contains plenty of sub-networks. Here we divide the evaluation indicators of health into three types, the physical health indicator, the mental health indicator and social health indicator. The consideration of ANNs are based on the principles shown as follows: i) set the assessment and prepared modules according to the part types, using the same indicators in the same ANN; ii) use different health indicators in different ANNs. This system can use 9 sub-networks consist of a series of indicators (physical indicators: physical symptoms and organ function, daily activity function and physical activity function; mental indicators: positive mood, mental symptoms and negative mood, recognition function; social health indicator: role activity and social adaption, social resources, social communication and social support ). In other words, the 9 sub-networks are made in parallel, corresponds to 3 evaluation and prepared modules respectively.

The identification and modeling of structure type. There are a large number of structure types of ANNs, according to the practical applications. Here, we choose the feed-forward structure, which is the so-called BP model. The input variables are health indicators and the output variables are the health level and sport prescription. Choosing suitable samples to train the ANN model, we can decide the weight coefficients. After training, the ANN model can evaluate the users with the input of input values. The assessment is the deduction process, which is the calculated output values after the input of known subjective values[3]. The learning pattern is the BP algorithm. It's main principle is: to a training sample, the inputted values can be calculated forward via the existing connection weights. After the interaction when passing each neurons, the output can be obtained. After that, the output values are compared with the expected output values. If there is any deviation (training error), the deviation can be transmitted in an opposite side[4]. The coefficient and thresholds can thus be adjusted in order to make the sample output as close as the expected output, which means that the price function of errors (optimization target function) $\mathrm{E}$ is the lowest. A BP network is shown in Figure 2:

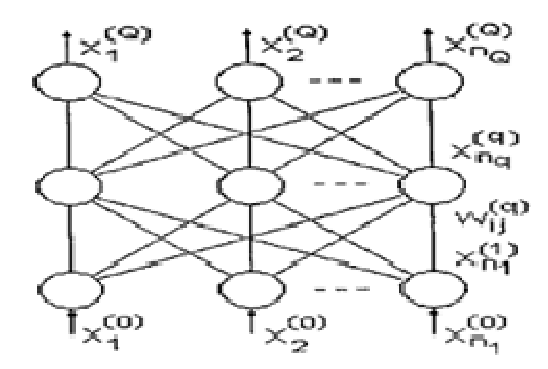

Fig. 2 Structure of a feed-forward neural network 
In the network shown in Figure 2, we set the number of neuron in the qth layer as nq. The connected weight coefficient from the q-1th layer, jth neuron to the qth layer, ith neuron is Wij(q), the transform equations of the input and output is:

$$
\begin{aligned}
\mathrm{S}_{\mathrm{i}}{ }^{(\mathrm{q})}=\sum_{\mathrm{j}=0}^{\mathrm{n}_{\mathrm{q}-1}} \mathrm{~W}_{\mathrm{i} j}{ }^{\mathrm{q}} \mathrm{X}_{\mathrm{j}}{ }^{(\mathrm{q}-1)} \quad\left(\mathrm{X}_{0}{ }^{(\mathrm{q}-1)}=\theta_{\mathrm{i}}{ }^{(\mathrm{q})}, \mathrm{w}_{\mathrm{j} 0}{ }^{(\mathrm{q})}=-1\right) \\
\mathrm{X}_{\mathrm{i}}{ }^{(\mathrm{q})}=f \quad \mathrm{~s}_{\mathrm{i}}{ }^{(\mathrm{q})}=\frac{1}{1+\mathrm{e}^{-\mu s i \mathrm{q})}}
\end{aligned}
$$

where $\mathrm{u}$ is the learning rate, $\mathrm{i}=1,2, \cdots, \mathrm{n}_{\mathrm{q}} \mathrm{j}=1,2, \cdots, \mathrm{n}_{\mathrm{q}-1} \mathrm{q}=1,2, \cdots, \mathrm{Q}$

In the learning process of BP algorithm, to reduce the errors, we usually use learning law. The price function of errors EP is:

$$
\mathrm{E}_{\mathrm{p}}=\frac{1}{2} \sum_{i}\left(\mathrm{t}_{\mathrm{pi}}-\mathrm{x}_{\mathrm{pi}}\right)^{2}
$$

where $\mathrm{P}$ is the sample number.

$\mathrm{t}_{\mathrm{pi}}, \mathrm{x}_{\mathrm{pi}}$ is the ideal output and actual output of the ith $\mathrm{p}$ sample, respectively.

To minimize the target function EP, gradient method is a typical method for solution. The correcting value of the weight is:

$$
\Delta \mathrm{W}_{\mathrm{ij}}{ }_{\mathrm{ij}}^{(\mathrm{q})}=\eta \delta_{\mathrm{Pj}}^{(\mathrm{q})} \mathrm{x}_{\mathrm{i}}{ }^{(\mathrm{q})}=-\eta \sum_{p=1}^{p} \frac{\partial \mathrm{E}_{\mathrm{p}}}{\partial \omega_{\mathrm{ij}}{ }^{(\mathrm{q})}} \quad \delta_{\mathrm{Pj}}^{(\mathrm{q})}=-\eta \frac{\partial \mathrm{E}_{\mathrm{p}}}{\partial \mathrm{S}_{\mathrm{i}}^{(\mathrm{q})}}
$$

where $\eta_{\text {is }}$ the gain factor, $\delta \mathrm{pj}^{(\mathrm{q})}$ is the training error of the jth neuron in the Qth layer.

\section{Health Evaluation and Prescription Constitution System Based on ANNs Combined with the Expert System}

\section{Existing Problems and their Solutions}

ANNs can make the self health evaluation and prescription constitution make the structure simple and clear, and meanwhile, it can adapt to the sudden change of emergency. However, there is still several disadvantages. First of all, in the complex society of users, health conditions usually change. Due to ANNs is faced to objects, it cannot adapt to these changes and may influence the evaluation results. Secondly, due to the extension and complexity of back-up protection, results are usually in a single aspect, rather than two aspects. Therefore, it cannot be used for the evaluation of the whole system. Apparently, these two issues are all involved in logical deduction, which is the advantage of expert system. Therefore, the combination of expert system and ANNs can keep the advantages of strong learning capacity, fast-parallel processing capacity and logical deduction at the same time. It is a good choice for health evaluation and prescription constitution.

\section{Introduction to Expert System}

Expert system is a computer programming system based on a large number of expertise and experimental knowledge, which can be used for a certain area. Using human experts' knowledge, experiences and methods for solutions can resolve the area's difficult problems, which can make deductions based on imprecise, uncertain or incomplete information and make conclusions. The system can make choices to the indicators according to users' subjective senses and diagnose, deduce and process, which cannot be precisely described by mathematical models and solved by traditional mathematical approaches. Therefore, the processing of expert system can suitably have this advantage. 


\section{Design of Expert System}

Principles of Design. To introduce the expert system to the self health evaluation and sport prescription, we should first see the system as a knowledge-based system, and thus the core (controller) should reflect the knowledge deduction mechanism. A expert system can be divided into two parts: the first part is the knowledge database, being used for express the expert's level in a certain concluded form. The second part is the deduction mechanism, deducing the problems and solutions based on the knowledge database and making judgments and decisions (based on various of numeric algorithms). The deduction process is shown in Figure 3. In addition, to control acquiring knowledge, knowledge expression (development of data models) and the design of database, principles of the consideration should be shown as follows: i) acquiring knowledge decides the performances of an expert system, but the acquisition of various knowledge should be controlled in a certain range; ii) expression of knowledge is the basis of an expert system. A suitable expression of self health evaluation and sport prescription should be chosen. A "Tree" structure of a database is very suitable for the self health evaluation and sport prescription; iii) The design of database should be based on the self health evaluation and sport prescription using a "Tree" model.

Design of Structure. The structure of self health evaluation and sport prescription based on ANNs and expert system is shown in Figure 3:

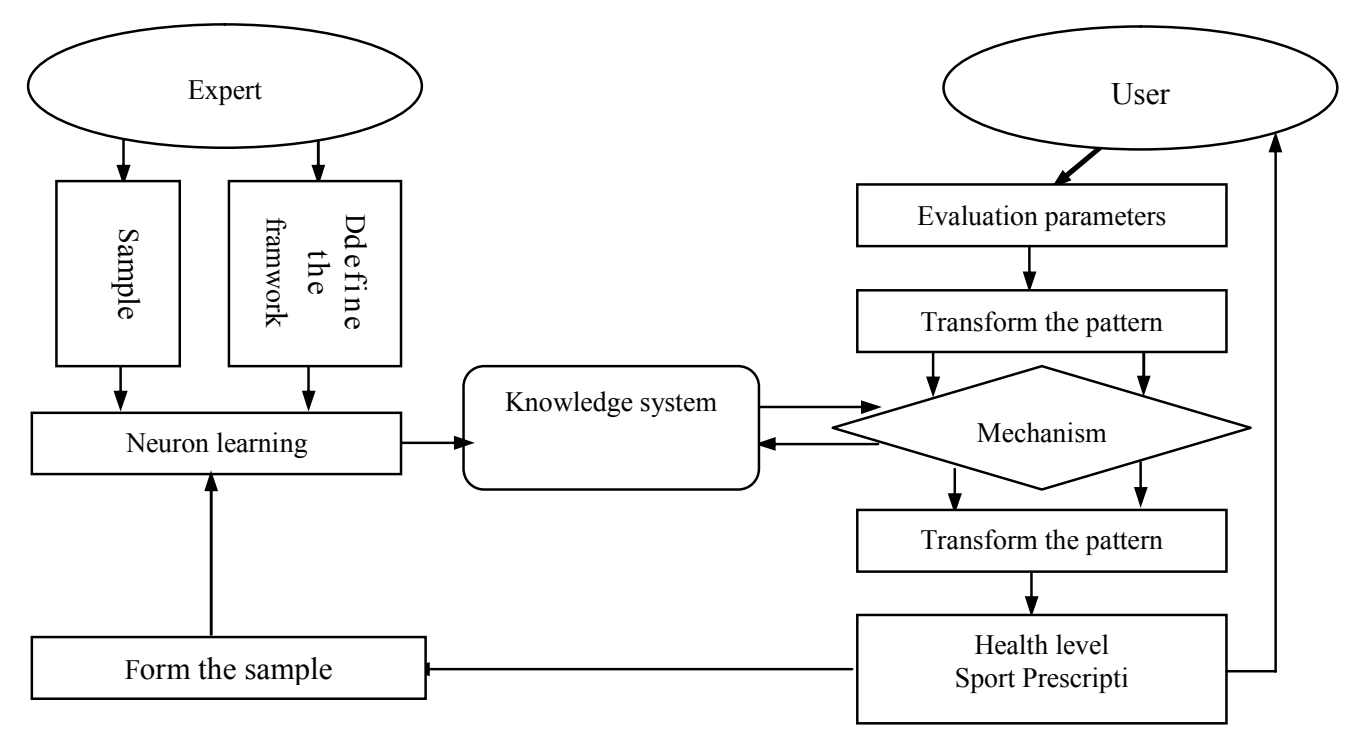

Fig. 2 The structure of self health evaluation and sport prescription based on ANNs and expert system

The system consists of two parts, the development environment and the runtime environment. The development environment consists of three parts, which can be shown by obtaining the knowledge database via learning in this example: i) learning sample; ii) define the framework of the system; iii) neuron learning. The runtime environment is actually the expert system, which can solve the actual problems, including i) self health evaluation parameters; ii) transformation of the input pattern; iii) mechanism deduction; iv) knowledge-based system (knowledge base) and v) transformation of the output pattern.

The learning of the expert system in the development environment is the adjustment of weights in accordance with errors of actual output values based on the experiences and examples and principles of expert system and BP networks. The runtime environment and practical applications are the process of transformation and association of memorized information, which response to the input of the system. Specifically, the system can give a series of health levels and sport prescriptions in accordance with each user's information, and store the process, which is set as the learning process of a sample. After doing exercises for several times, users can reenter the system and process the measurements, adjusting the prescription. During this adjusting process, this system can adjust the 
plan according to the exercising effects, improving the rationality of the contents. At the same time, the contents of knowledge database will be revised. When a new user enters the system, if he/she uses the same example knowledge, the system can give out a more reasonable prescription after revisions. That is to say, the system develops a file for every old user, and make more scientific health levels and sport prescription.

\section{Conclusion}

Here, we assume that in a self health evaluation and sport prescription system based on ANNs and expert system, the ANN is the core of evaluation and prescription, while the expert system is the necessary supplement to the disadvantage of the ANN, which both absorb the two principles' advantages. This system can offer an important reference basis to people who is pursuing healthy sport excising or being a patient. We can assume that if the system can be extended into the Internet, it would be great significative to promote the civil fitness and improve the civil body constitutions.

\section{References}

[1] H. Li, W. Leng, Y. Zhou, F. Chen, Z. Xiu, D. Yang, Evaluation models for soil nutrient based on support vector machine and artificial neural networks, The Scientific World Journal, 2014 (2014) 478569.

[2] Xiao D, Chunling G, sport law, third ed., Law Press China, Bei Jing, 2006.

[3] D. Yang, H. Li, C. Cao, F. Chen, Y. Zhou, Z. Xiu, Analysis of the oil content of rapeseed using artificial neural networks based on near infrared spectral data, Journal of Spectroscopy, 2014 (2014) 901310.

[4] J F. Wang, B Zhang, Study on New Adaptive Complex Equalizers Con-structde by Radial Basis Function Neural Networks, Systems Engineering and Electronics. 25(2003) 848 - 859. 